\title{
Theory of the hatchet planimeter
}

\author{
SIMON FARTHING \\ Department of Mathematics, Simon Fraser University, Burnaby B C, V5A 1S6, Canada \\ Present address; 975 Tuam Road, RR 1 Sidney B C, V8L 3R9, Canada
}

MS received 12 June 1984; revised 13 May 1985

\begin{abstract}
The remarkable area measurement property of the hatchet planimeter is proved directly and easily by a series expansion. The previously known correction terms are readily generated and extended by a new analysis which now easily shows any blade misalignment to be of minor effect. This allows rationalizing the design for adjustment to give the best precision for the size of the figure. A new operating scheme, illustrated by an example, attempts to ensure accuracy at the minimum of user sophistication and calculation, to realize the hatchet planimeter's enormous advantage in cost, mechanical simplicity and ruggedness over the Amsler planimeter. This is the background to an attempt to popularize this planimeter for rural engineering (Farthing et al 1985).
\end{abstract}

Keywords. Area; planimeter; hatchet; Prytz.

\section{Introduction}

The standard analog instrument for measuring irregularly shaped areas, such as those enclosed by contours on a map, curves on a plan, or underneath a graphed function, is the polar planimeter invented by Jakob Amsler in 1856. In it a wheel vernier-graduated to 0.001 of a turn - is moved obliquely to its axis, so that it partly slips over the paper and partly rotates. This necessitates a delicate needle point bearing in an exacting alignment, and a fine worm gearing to count complete revolutions; explaining the $£ 165$ starting price in 1982.

In stark mechanical and price contrast is the hatchet planimeter of no moving parts at all. It was in 1886 (Willers 1926) that Captain Andreas Prytz made the first one by bending an iron rod twice and working one end into a pointer $P$ and the other into a rockered knife edge $K$, as in figure la. Holding this giant staple upright at $P$, he moved $P$ around the contour of an area with the knife edge zigzagging over the paper in pursuit. When he measured the net arc $K_{0} K_{1}$ between the start and finish positions of $K$, he found that its product with the much larger distance $P K$ gave the area quite accurately.

This product can be interpreted as the area $K_{0} K_{1} P^{\prime} P_{0}$ in figure 1 obtained by drawing the arc $K_{0} K_{1}$ about $P_{0}$ and the arc $P_{0} P^{\prime}$ about $K_{1}$, with $K_{1} P^{\prime}$ parallel to $K_{0} P_{0}$. In fact the area circumnavigated by $P$ was proved by Willers (1926) using his general planimeter theorem (Willers 1948) to be exactly equal to the shaded area in figure 1 , which is close to $K_{0} K_{1} P^{\prime} P_{0}$.

However this identity does not help much in understanding how best to minimize or correct the inaccuracy of Prytz's approximate formula. For this Willers (1926) quotes the result of an analysis by Runge; the proof for a very similar infinite series by Hill (1894) is very laborious. More befitting the utter physical simplicity of the device is the following new analysis in terms of the rest of figure $1 \mathrm{~b}$. 


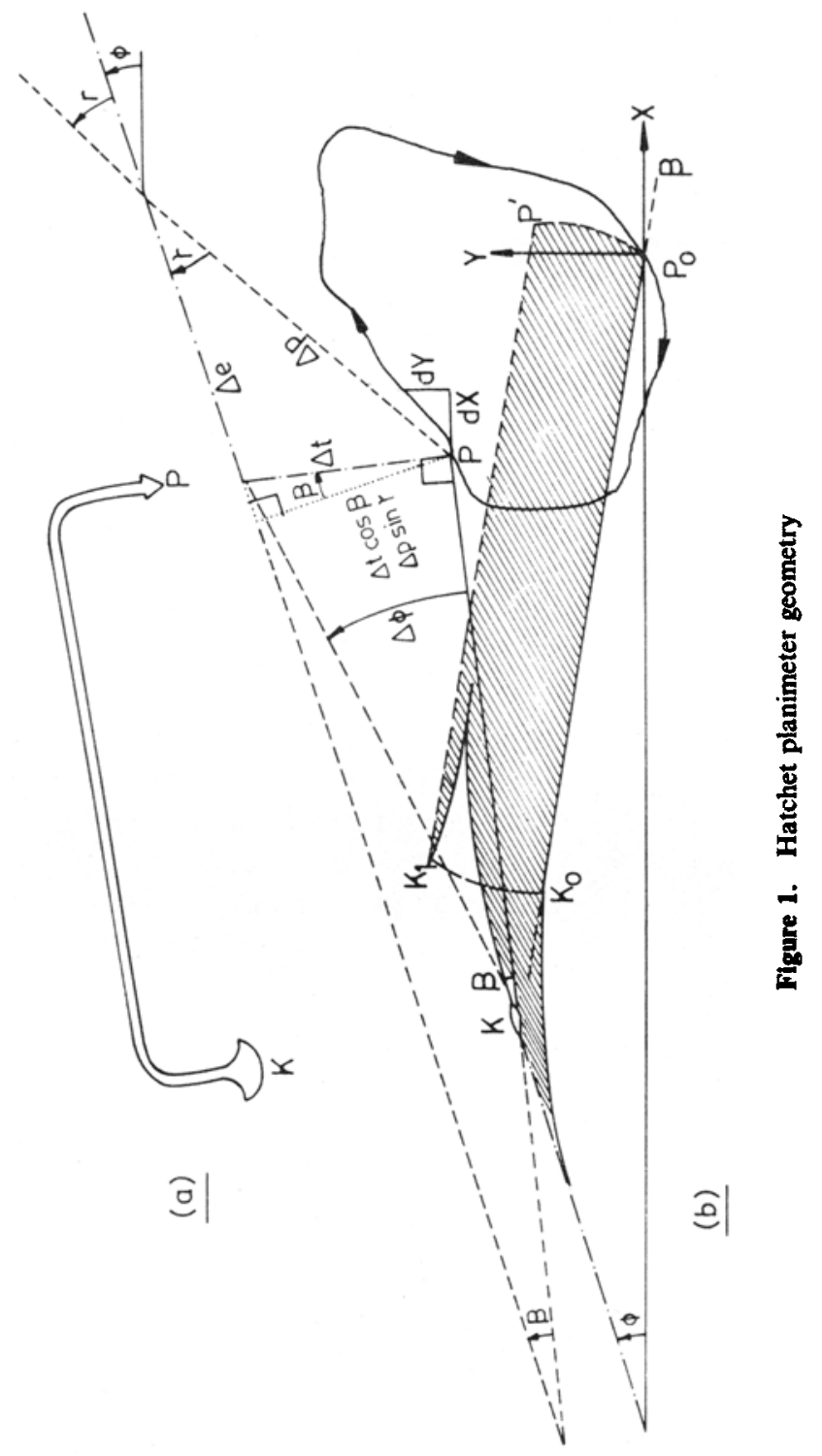




\section{Theory}

\subsection{Equation for the angle of rotation}

The 'bite' of the knife edge on the paper prevents it moving sideways so that it can only move forward or rotate about its centre of contact $K$. Let $\phi$ be the inclination of the blade to its horizontal starting orientation, taken as the $X$ direction in an $X, Y$ coordinate system with the origin at the starting point of the pointer $P$ at ' $P_{0}$ '. Offset of $P$ from the line of the knife edge is represented by the angle $\beta$, e.g. of $P_{0} K_{0}$ to the $X$ axis.

Any small displacement $\Delta p$ of the pointer $P$ at any angle $\gamma$ to the edge can be broken down into two steps. First $P$ is rotated about $K$ through a small angle $\Delta \phi$ and so displaced perpendicular to $P K$ by $\Delta t=P K \Delta \phi$. Secondly the whole planimeter is translated $\Delta e$ at constant $\phi$, in the direction of the edge and so at angle $\beta$ to $P K$. Similarly $\Delta t$ is $\beta$ off perpendicular to the edge; therefore the component of movement so perpendicular is $\Delta t \cos \beta$ as well as $\Delta p \sin \gamma$.

$$
\cos \beta P K \Delta \phi=\Delta p \sin \gamma .
$$

Then resolving an infinitesimal displacement of $P$ along the contour of the area as a movement $\mathrm{d} p=\mathrm{d} X$ in the $X$ direction with $\gamma=-\phi$ and then, $\mathrm{d} p=\mathrm{d} Y$ with $\gamma=\pi / 2-\phi$ in the $Y$ direction, the net $\mathrm{d} \phi$ gives what might be called the hatchet planimeter law:

$$
\cos \beta P K \mathrm{~d} \phi=-\sin \phi \mathrm{d} X+\cos \phi \mathrm{d} Y \text {. }
$$

\subsection{Series expansion}

When $b$ the maximum dimension of the figure is much less than $P K$, (2) shows $\phi$ will be of small order $b / 2 P K$. Then the sin and cos on the RHs could be profitably expanded in powers of this small angle, and a series solution in powers of $b / 2 P K$ can be found.

Formally non-dimensionalize $X$ and $Y$ by b/2 defining $x=2 X / b, y=2 Y / b$ and define the small non-dimensional parameter $\varepsilon=b /(2 P K \cos \beta)$. Then dividing (2) by $P K$ and expanding $\cos \phi$ and $\sin \phi$,

$$
\mathrm{d} \phi=\varepsilon\left(1-\frac{1}{2} \phi^{2}+\frac{1}{24} \phi^{4} \ldots\right) \mathrm{d} y-\varepsilon\left(\phi-\frac{1}{6} \phi^{3}+\frac{1}{120} \phi^{5}+\ldots\right) \mathrm{d} x,
$$

which solve by expanding $\phi=\varepsilon \phi_{1}+\varepsilon^{2} \phi_{2}+\varepsilon^{3} \phi_{3}+\ldots$ Substituting and equating coefficients of equal powers of the arbitrary parameter $\varepsilon$ will give $\mathrm{d} \phi_{i}$ on the LHSin terms of the lower order $\phi_{i}$ and $d y$ and $d x$ on the RHS. The prime interest here is in the net change $\Delta \phi_{i}$ in a circuit of the perimeter in which $P$ returns to the origin, and the main tool is integration by parts in $x$ and $y$ since their end values are always equal at zero, and the derivatives of the $\phi_{i}$ on the RHs, known from previous orders. Consider all contour integrations clockwise.

\subsection{The first two terms and the blade design}

Equating terms in $\varepsilon$ and $\varepsilon^{2}$ on both sides of (3) gives

$$
\begin{aligned}
& \mathrm{d} \phi_{1}=\mathrm{d} y, \therefore \phi_{1}=y \text { and } \mathrm{d} \phi_{2}=-\phi_{1} \mathrm{~d} x=-y \mathrm{~d} x, \\
& \therefore \Delta \phi_{2}=-\oint y \mathrm{~d} x=-\oint \mathrm{d} y \mathrm{~d} x=-a .
\end{aligned}
$$

Thus $\phi_{1}$ returns at the end of the trace to its starting value but $-\phi_{2}$ finishes as the non- 
dimensional area $a$ of the figure. This $\phi$ oscillation as $\varepsilon y$ during the trace with only a small outcome $\varepsilon^{2} a$ necessitates accurate realization of the ideal knife edge behaviour. Lateral slippage must be reduced far below visible levels before the path of $K$ is retraced as it should be when $P$ motion is reversed. Such reversal doesn't check exactly for the original Prytz design with its mild steel hatchet edge (Satterly 1921). However, the mass produced stainless steel scalpel blade approaches perfect reproduction even on hard drawing film if loaded to say $200 \mathrm{G}$ (Farmer 1978). The weight required is never so much that the blade's movement leaves an impression, let alone cut the paper. But it does cause the blade to drag enough that the paper must be tightly taped down to stay still and flat (a rotating blade Willers (1926) to eliminate the drag would be a custom item expensive to make to the high hardness required, and its bearing would have to be very precise not to have lateral play which is effective slip). A simpler way to reduce the drag and so make the tracing easier and smoother is to take down a piece of wax paper for the blade to slide on. Also when the blade is pushed in to mark $K_{0}$ and $K_{1}$, the wax paper goes white at the dents or 'kerfs', so they become very clear against a dark underlay.

\subsection{Higher order correction terms}

Equating powers of $\varepsilon^{3}$ on each side of (3) gives

$$
\begin{aligned}
& \mathrm{d} \phi_{3}=-\frac{1}{2} \phi_{1}^{2} \mathrm{~d} y-\phi_{2} \mathrm{~d} x, \\
& \therefore \mathrm{d} \phi_{3}=-\frac{1}{2} y^{2} \mathrm{~d} y-\mathrm{d}\left(x \phi_{2}\right)+x \mathrm{~d} \phi_{2} .
\end{aligned}
$$

Substituting for $\mathrm{d} \phi_{2}$ from (4), $-\Delta \phi_{3}$ is the $x$ moment of the area $a \bar{x}$. Next,

$$
\mathrm{d} \phi_{4}=-\frac{1}{2}\left(2 \phi_{1} \phi_{2}\right) \mathrm{d} y-\left(\phi_{3}-\frac{1}{6} \phi_{1}^{3}\right) \mathrm{d} x .
$$

Two successive integrations by parts around the contour again puts the $\phi$ terms on the RHS into differentials known from previous rounds, leaving,

$$
\Delta \phi_{4}=-\oint \frac{1}{6} y^{3} \mathrm{~d} x+\oint \frac{1}{6} x^{3} \mathrm{~d} y=-\frac{1}{2} \oint\left(x^{2}+y^{2}\right) \mathrm{d} x \mathrm{~d} y=-\frac{1}{2} a r^{2},
$$

where $r$ is the non-dimensional polar radius of gyration of the area about the starting point. Finally the fifth order problem from (3) is

$$
\mathrm{d} \phi_{5}=\left(-\frac{1}{2} \phi_{2}^{2}-\frac{1}{2} 2 \phi_{1} \phi_{3}+\frac{1}{24} \phi_{1}^{4}\right) \mathrm{d} y-\left[\phi_{4}-\frac{1}{6}\left(3 \phi_{1}^{2} \phi_{2}\right)\right] \mathrm{d} x .
$$

Again integrating by parts in $x$ and $y$ to exploit the known differentials of the previous $\phi$ 's leads after 4 rounds to

$$
\Delta \phi_{5}=\frac{1}{2} \oint y^{2} \phi_{2} \mathrm{~d} x+\oint \frac{1}{24} x^{4} \mathrm{~d} y=-\frac{1}{2} \oint y^{2} \mathrm{~d} x \int y \mathrm{~d} x-\oint \frac{1}{6} x^{3} \mathrm{~d} x \mathrm{~d} y .
$$

As the algebra is increasing rapidly, it is fortunate that the above terms provide as much information as can practically be used. The 1 st term in $\phi_{5}$ is also the first not to be reducible to a single contour integral of powers of $x$ and $y$.

In practice the angle $\Delta \phi=\varepsilon^{2} \Delta \phi_{2}+\varepsilon^{3} \Delta \phi_{3}+\ldots$ is inconvenient to measure, especially with adjustable $P K$. More amenable is the chord $K_{0} K_{1}$, call it $H$, which gives $\Delta \phi=-2 \sin ^{-1}(H / 2 P K)$, and upon expanding in terms of $h=4 H \cdot P K \cos ^{2} \beta / b^{2}$ to get a matching second order term to lead

$$
-\Delta \phi=2 \sin ^{-1}\left(\varepsilon^{2} h / 2\right)=\varepsilon^{2} h+\varepsilon^{6} h^{3} / 24+\ldots
$$


So then equating this expansion and the previous one for $-\Delta \phi$, i.e.,

$$
\begin{aligned}
-\Delta \phi= & \varepsilon^{2} a+\varepsilon^{3} a \bar{x}+\frac{1}{2} \varepsilon^{4} a r^{2}+\varepsilon^{5}\left[\oint \frac{1}{2} y^{2} \mathrm{~d} x \int y \mathrm{~d} x+\oiint \frac{1}{6} x^{3} \mathrm{~d} x \mathrm{~d} y\right] \\
& +\ldots \\
= & \varepsilon^{2} h+\varepsilon^{6} h^{3} / 24+\ldots
\end{aligned}
$$

Factoring $a$ and dividing through by $\varepsilon^{2}$

$$
h=a\left(1+\varepsilon \bar{x}+\frac{1}{2} \varepsilon^{2} r^{2}+\varepsilon^{3}\left[\oint \frac{1}{2} y^{2} \mathrm{~d} x \int y \mathrm{~d} x+\oint \frac{1}{24} x^{4} \mathrm{~d} y\right] / a+0\left(\varepsilon^{4}\right) \ldots\right),
$$

or converting back to dimensional terms by multiplying by $\frac{1}{4} b^{2}$, defining $S=P K \cos \beta$,

$$
\begin{aligned}
& H S \cos \beta=A\left(1+\bar{X} / S+\frac{1}{2} r^{2}(b / 2)^{2} / S^{2}\right. \\
& \left.+\left[\oint \frac{1}{2} Y^{2} \mathrm{~d} X \int Y \mathrm{~d} X+\frac{1}{6} \oiint X^{3} \mathrm{~d} X \mathrm{~d} Y\right] / S^{3} \oiint \mathrm{d} Y \mathrm{~d} X+\ldots\right) .
\end{aligned}
$$

\section{Application}

\subsection{Best tracing strategy}

So the first correction to Prytz formula $A=H S \cos \beta$ is due to the moment $\operatorname{arm} \bar{X}$ (scaled relative to $S$ ) of the area about the $Y$ axis (which was defined as passing through $\boldsymbol{P}_{0}$ normal to the blade's initial orientation). Thus one should start $\boldsymbol{P}$ near the figure's line of balance perpendicular to the knife edge, as will help make the shaded true area under the knife's path be accurately approximated by Prytz's $P K \cdot K_{0} K_{1}$ or the area $K_{0} K_{1} P^{\prime} P_{0}$ in figure 1 . Judging the balance is easiest in the area's most symmetrical and narrowest dimension, to which the planimeter should be thus be set parallel. Note that if the same trace is repeated but starting with the planimeter on the other righthand (R) side of the line of balance, any residual $\bar{X} / S$ interference would be reversed as would the 3rd order integral term as well ( $X$ replaced by $-X$ top and bottom) in the two cases of (9). Thus averaging these equations changes (9) to give $A$ in terms of the average $\vec{H}=\left(H_{R}+H_{L}\right) / 2$, free of the 1 st and 3rd correction terms (Willers 1926).

What will always remain is the second order error due to the mean square distance $r^{2}$ (nondimensional) of the figure about the start/finish point $\boldsymbol{P}_{0}$. By the parallel axis theorem this is minimized by choosing $P_{0}$ to also lie roughly on the line of balance of the figure parallel to the planimeter, i.e., at the centroid of the figure where all balance lines intersect. In naval architecture the centroid is already found for other purposes by balancing a piece of stiff paper cut out to the area's shape. Then to trace around the area one must move out to the perimeter along a 'cut', circle the perimeter and then return to the interior starting point along exactly the same cut.

In the final term retained in the expansion the $x^{3}$ integration should be small because of the $\frac{1}{6}$ factor and $x$ being chosen in the direction of the shorter dimension of the figure. In the other more major term, $\frac{1}{2} y^{2} \mathrm{~d} x$ vanishes along a cut in the $Y$ or $X$ direction, and around the perimeter oscillates, and taken alone its integral $\iint y \mathrm{~d} x \mathrm{~d} y$ would vanish 
since the starting point is at the centroid. Its multiplier $\phi_{2}$ begins building during the outer trace as the accumulation of area between the arc traversed and the $x$ axis. So that this growing multiplier interferes as little as possible with the cancellation of $\frac{1}{2} y^{2} \mathrm{~d} x$, that cancellation should be spread over the trace as uniformly as possible by having $\mathrm{dx}$ change signs as many times as possible. This means taking the cut in the $Y$ direction so that dx changes sign 3 times rather than in the $X$ direction where it changes sign only once. For instance for a square $4(2)^{1 / 2} \Delta \phi_{5}=-\frac{1}{8}$ for the $Y$ cut vs 2 for the $X$ cut. However, in practice this theoretical advantage of having the cut perpendicular to the planimeter's original placement seems negated by the tendency of the mainly rotational initial blade motion involved to be distorted by the starting kerf in the paper.

A more important new result is the influence of misalignment $\beta$ of the blade with the planimeter arm, as experimentally verified at $\beta=45^{\circ}$. For smaller unintentional misalignments, the correction is second order, only $3 \%$ at $\beta=10^{\circ}$, and easily calibrated in any case, in good contrast to the lst order alignment error in polar planimeters, which can only be neutralized by a compensating design allowing a second trace. An immediate application of the hatchet planimeter's tolerance for misalignment is to offset the pointer $\boldsymbol{P}$ from a long main beam so that it can be attached at the optimum $P K$ for the particular area required.

\subsection{Optimum length $P K$}

The other source of error besides Prytz's approximate formula is the uncertainty of say $+0.1 \mathrm{~mm}$ in measuring $H$. The eye also limits the trace to the same accuracy so any planimeter is uncertain to a fraction $0\left(0.1 \mathrm{~mm} / \frac{1}{2} b\right)$ in the area, but the above contribution is $0\left[0.1 \mathrm{~mm} P K /(b / 2)^{2}\right]$ which is bigger by $2 P K / b$ or $\varepsilon^{-1}$. Thus if there is any choice, an area should be drawn at large scale to suit the Prytz device. For a given size of figure there will be an optimum $\varepsilon$ at the best length $P K$ for that scale. Suppose the improved calculating formula for $\beta=0, S=P K, \bar{X} \approx 0$ :

$$
A=H \cdot S\left(1-\frac{1}{8} r^{2} b^{2} / S^{2}\right) \pm 0.01 \mathrm{~cm} \cdot S \pm \frac{1}{8} \Delta r^{2} b^{2} H / S \pm H \Delta \bar{X}
$$

is used, where $r^{2}(b / 2)^{2}$ is an estimate of the polar second moment of the area with $\Delta r^{2}$ the uncertainty in the nondimensional gyration $r^{2} . r^{2}$ and $\Delta r^{2}$ are judged by comparing the shape of the area with dumbell $r^{2}=1$, circle $r^{2}=0.5$, rectangle and equilateral triangle $r^{2}=0 \cdot 33$, semicircle $r^{2}=0 \cdot 32$, very eccentric ellipse $r^{2}=0.25$, and finally very squat isosceles triangle or a flat diamond $r^{2}=0.17$.

To get an $a$ priori estimate of the best $S$ on the basis of only $b$, it will be assumed that the uncertainty in half the polar inertia will be bounded by $\pm 0075 \pi(b / 2)^{4}$, i.e., $r^{2}$ accurate to $\Delta r^{2}=0-15$ when the area is at its maximum relative to $(b / 2)^{2}$ of $\pi$ for a circle. Then minimizing the sum of this uncertainty as divided by $S^{2}$ in the above equation and the $H$ measurement uncertainty giving $\pm(0.01 \mathrm{~cm}) S$ there, with respect to $S$ gives $S_{\text {opt }}=30.86 \mathrm{~cm}\left(\frac{1}{10} b \text { in } \mathrm{cm}\right)^{4 / 3}$. At which the net uncertainty would be a minimum of $\frac{3}{2}(0.1 \mathrm{~mm}) S_{\text {opt }}=0.463 \mathrm{~cm}^{2}\left(\frac{1}{10} b \text { in } \mathrm{cm}\right)^{4 / 3}$. The final error term in (10) due to the uncertainty $\Delta \bar{X}$ in estimating the centroid can always be eliminated by a second trace if it is unacceptable. For a given $b, H \leqslant \pi(b / 2)^{2} / S$, so the $\Delta \bar{X}$ term will always be less than half the above minimum uncertainty if $\Delta \bar{X}<0.91 \mathrm{~mm}\left(\frac{1}{10} b \text { in } \mathrm{cm}\right)^{2 / 3}$, quite a strict criterion. These results are summarized for different $b$ for ready practical reference in table 1. Definitive assessment of the accuracy when needed can be made $a$ posteriori by calculating the \pm terms in (10) at the same time as the answer. 
Table 1. A priori adjustment of the hatchet planimeter to the best accuracy.

\begin{tabular}{|c|c|c|c|c|c|c|}
\hline Figure max dimension $b$ in $\mathrm{cm}$ & 5 & 7 & 10 & 15 & 20 & 25 \\
\hline Optimum setting $S=P K$ in $\mathrm{cm}$ & $12 \cdot 2$ & $19 \cdot 2$ & 31 & 53 & 78 & 105 \\
\hline Maximum uncertainty $\Delta A$ in area $A$ in $\mathrm{cm}^{2}$ & 0.18 & 0.29 & 0.46 & 0.80 & $1 \cdot 17$ & $1 \cdot 57$ \\
\hline$\Delta \bar{X}$ of centroid in $\mathrm{mm}$ for single trace only & & & & & & \\
\hline increasing $\Delta A$ by less than $50 \%$ & $0-57$ & 0.72 & 0.91 & $1 \cdot 2$ & $1 \cdot 4$ & $1 \cdot 7$ \\
\hline
\end{tabular}

\subsection{Practical Design}

Figure 2 shows the scalpel blade clamped to the edge of a meter stick with a tack through its slot and one on its top edge directly above the point of contact. An inch wide yardstick is very stiff laterally so that sideways buckling which would change $\beta$ in proportion to the push or pull from $P$ and cause irreversibility is minimized. From a discarded perspex ruler a cursor can be made with the end of one of the graduations serving as point $P$. This $P$ is located say $1 \mathrm{~cm}$ outside the edge to which the blade is attached, by countersinking screws from the perspex through a $6 \mathrm{~mm}$ spacer block into the middle of the meter stick at the distance given by table 1 . This flat, say $4 \mathrm{~cm} \times 2 \mathrm{~cm}$, piece of perspex also stabilizes the elevated meter stick to allow pausing during the trace.

\subsection{Example}

To illustrate the procedures and formulae recommended above and to provide a test case that a reader can reproduce with his newly constructed planimeter, an isosceles triangle of base $20 \mathrm{~cm}$ and side $17.5 \mathrm{~cm}$ was drawn. A move to a more humid environment and possibly stretching during the taping down unfortunately changed its exact dimensions to $20.07 \pm 0.01 \mathrm{~cm}$ base by $14.40 \pm 0.01 \mathrm{~cm}$ height so that the area was really $144.50 \pm 0.17 \mathrm{~cm}^{2}$. Following the table the perspex cursor was screwed in at about $78 \mathrm{~cm}$ from the blade; and to illustrate the centroid error, the starting point was arbitrarily placed at $6.00 \pm 0.01 \mathrm{~cm}$ from the base along the perpendicular bisector, the centroid really being $4.80 \mathrm{~cm}$ along.

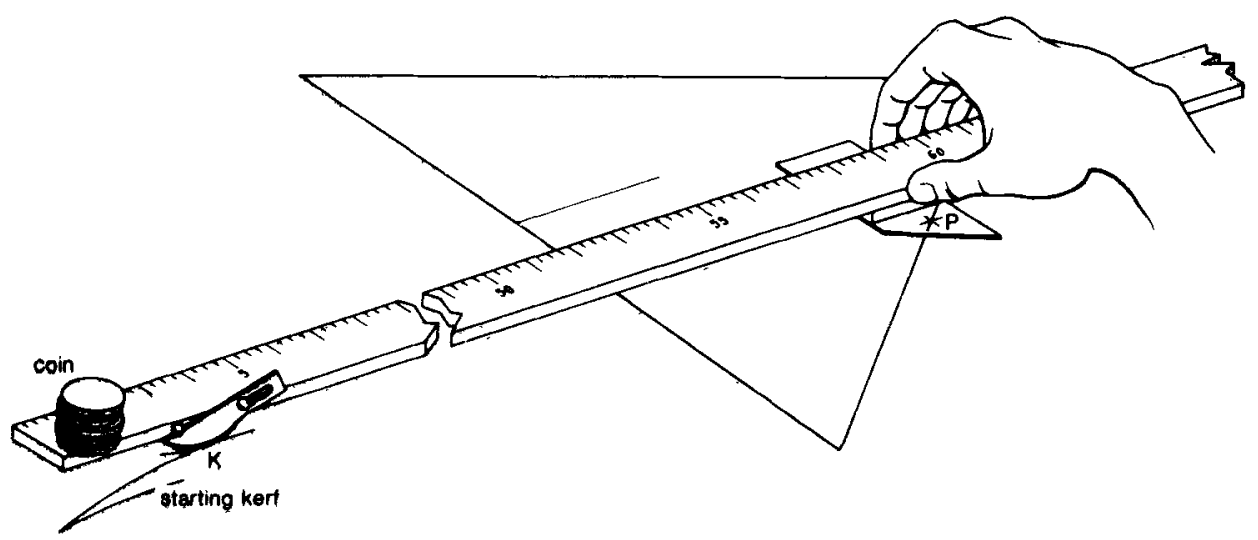

Figure 2. Practical design 
Then the planimeter/yardstick was placed down to the left crossing the base and on top of the perpendicular bisector so that $\Delta \bar{X}$ was negative. Pressing the blade in, then tracing along the bisector to the base, counter-clockwise around the triangle and then back along the bisector and finally pressing in again gave $H_{L}=1.81 \pm 0.01 \mathrm{~cm}$. Moving the blade beyond the starting kerf to glide over the virgin bit of wax paper during the trace reversed clockwise gave $H_{L}=1.86 \pm 0.01 \mathrm{~cm}$. The difference is attributed to minute blade slippage, in opposite sideways directions for opposite pointer motions. So Satterly's (1921) advice to use the average of the two seems wellfounded; here $\bar{H}_{L}$ $=1.835 \pm 0.01 \mathrm{~cm}$.

Then the drawing of the triangle was rotated through about $180^{\circ}$ to reverse the sign of $\Delta \bar{X}$, and retaped. Starting the blade from a new dent and performing the same counterclockwise trace as before gave $H_{R}=1.88 \pm 0.01 \mathrm{~cm}$, and on reversing clockwise $H_{R}=1.92 \pm 0.01 \mathrm{~cm}$. Thus $\bar{H}_{R}=1.90 \mathrm{~cm}$, a change of $0.065 \pm 0.02 \mathrm{~cm}$ agreeing with the $2 H \Delta \bar{X} / S=2 \times 1.86 \times 1.2 / 78=0.057$ expected from (9). Thus the grandmean $H$ to use in (10) was $1.8675 \pm 0.01 \mathrm{~cm}$, and $r^{2}$ was estimated as $0.30 \pm 0.03$ for a not-quiteequilateral triangle. Finally $S$ was measured as the distance from the centre of the kerfs to the starting point as $77.75( \pm 0.02) \mathrm{cm}$. So (10) gives in $\mathrm{cm}^{2}$,

$$
\begin{aligned}
A= & 1.8675 \times 77.75 \times\left[1-\frac{1}{8} \times 0.3(20.07 / 77.75)^{2}\right] \pm 0.01 \times 77.75 \\
& \pm \frac{1}{8}(0.03) \times(20.07)^{2} \times 1.8675 / 77.75
\end{aligned}
$$

Thus $A$ is predicted to be 144.84 or $0.34 \mathrm{~cm}^{2} \sim \frac{1}{4} \%$ above the true value, well within the combined uncertainty in the two values of $(0.78+0.03)+0.17 \sim 1 \mathrm{~cm}^{2}$.

Note that the difference in reversing is virtually the same for the left and right traces so that it appears that the second trace to nullify any slippage can be combined with the repeat trace usually needed because the centroid is hard to judge to the accuracy

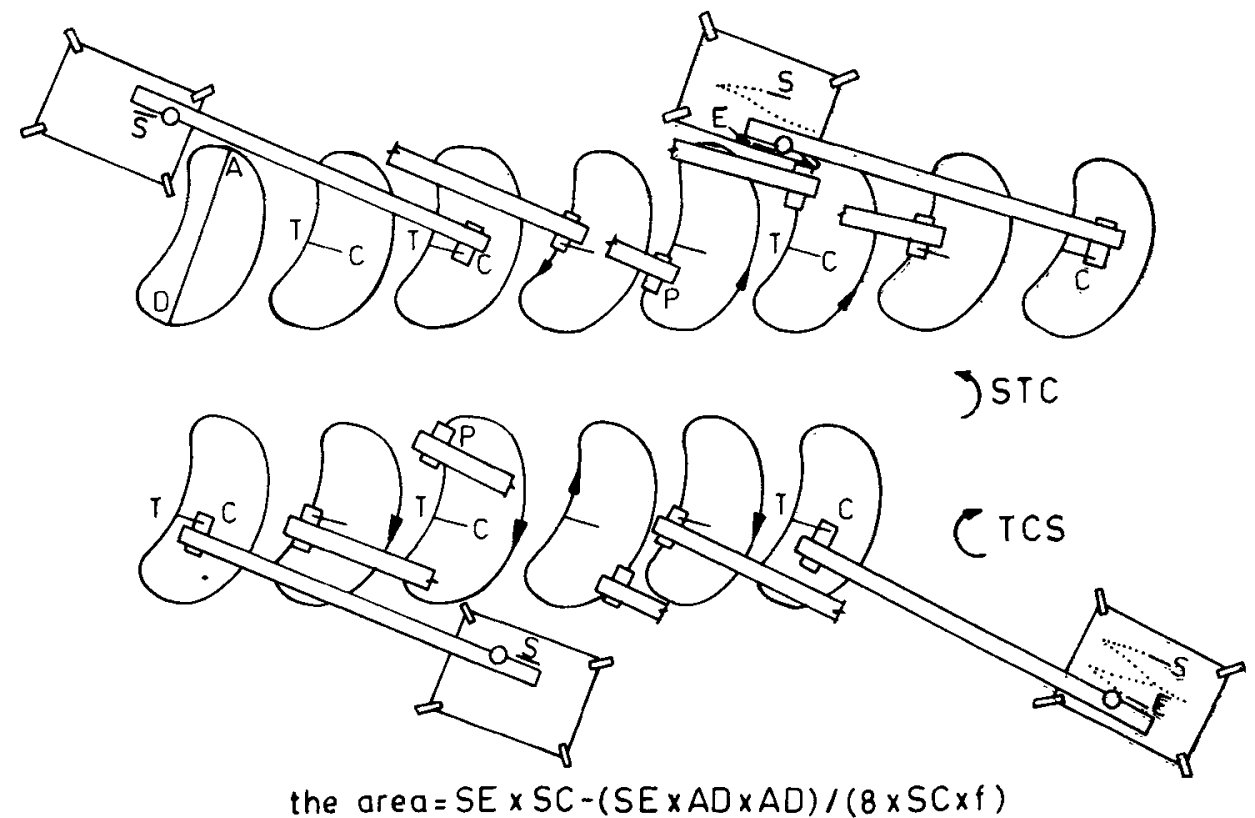

Figure 3. Summarized procedure for measuring the area (from Farthing et al 1985) 
required in table 1 . That is, trace from the left counterclockwise and then from the right clockwise. Here this would have given a good mean $H$ of $1.865 \pm 0.01 \mathrm{~cm}$. in just two traces.

Figure 3 (after Farthing et al 1985) summarizes the procedure developed above putting the calculating formula (10) directly in Euclidean length notation, with $f$ the 'guestimate' of $1 / r^{2}$, and with $S E$ understood to be the average of the two such chords in the depiction of the recommended traces.

The support of Gifford \& Partners, Southampton, U.K. and of Simon Fraser University for this work is gratefully acknowledged.

\section{References}

Farmer W 1978 Natl. Fisherman May: 54-55

Farthing S, Owen R, Gage S 1985 Appropriate Technology 12(2): 10-11

Hill F W 1894 Philas. Mag. 38: 265-268

Satterly J 1921 The Hatchet Planimeter, Univ. of Toronto Studies, Mathematical Series no. 2, pp. 3-24

Willers F A 1926 Mathematische Instnmente (Berlin: Springer-Verlag) soc. 13, pp. 82-85

Willers F A 1948 Practical analysis (New York: Dover) sec. 17, pp. 191-195 